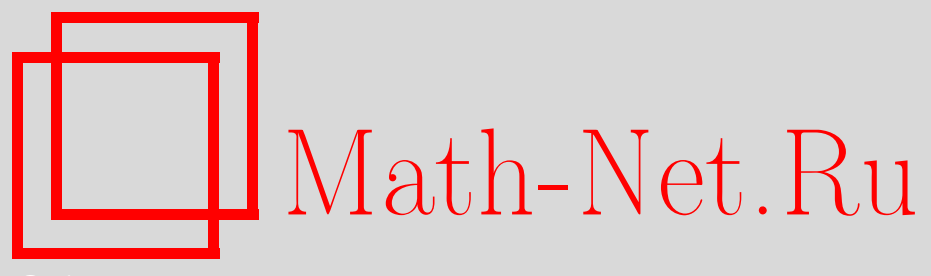

В. М. Демиденко, Критерий смежности вершин политопов, порождаемых подмножествами симметрической группы, Матем. заметки, 2006, том 80, выпуск 6, 838855

DOI: https://doi.org/10.4213/mzm3361

Использование Общероссийского математического портала Math-Net.Ru подразумевает, что вы прочитали и согласны с пользовательским соглашением http://www . mathnet.ru/rus/agreement

Параметры загрузки:

IP: 54.172 .240 .79

26 апреля 2023 г., 02:50:48

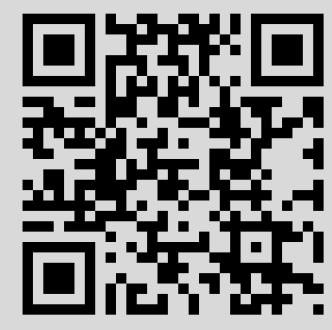




\title{
КРИТЕРИЙ СМЕЖНОСТИ ВЕРШИН ПОЛИТОПОВ, ПОРОЖДАЕМЫХ ПОДМНОЖЕСТВАМИ СИММЕТРИЧЕСКОЙ ГРУППЫ
}

\section{В. М. Демиденко}

\begin{abstract}
Предложена характеризация смежности вершин в классе подстановочных политопов, порождаемых произвольными подмножествами симметрической группы. Указанному классу принадлежат политопы известных классических задач о назначениях, 2- и 3-сочетаниях, коммивояжере и их различных модификаций. До настоящего времени вопросы, связанные со смежностью вершин, исследовались в основном для отдельных политопов. В данной работе для подстановочного политопа общего вида получены необходимые и достаточные условия (не)смежности его вершин, которые сформулированы в терминах подстановок и разрешимости специального вида систем линейных уравнений. Известные критерии смежности вершин политопа задачи о назначениях являются простыми следствиями предложенных условий. На основе последних разработана общая алгоритмическая схема для распознавания смежности вершин подстановочного политопа общего вида и оценена ее трудоемкость.
\end{abstract}

Библиография: 20 названий.

1. Введение. Выявление достаточно быстро проверяемых условий смежности вершин политопов напрямую связано с их полиэдральным описанием, а также с разработкой эффективных алгоритмов, осуществляющих обходы вершин граф-остовов политопов, с целью нахождения приемлемых решений соответствующих оптимизационных задач. $\mathrm{K}$ алгоритмам такого типа относятся симплексные процедуры и процедуры локального поиска. Для последних эффективно проверяемый критерий смежности вершин политопа является универсальным правилом "выхода" из тупиковой ситуации (достижение локального оптимума), применение которого в конечном итоге приводит к нахождению глобального оптимума.

Обширный список литературы по исследованию остовов в основном трехмерных политопов представлен в монографии Грюнбаума [1]. В обзоре Хаусмана [2] приводятся интересные теоретические результаты о смежности вершин политопов, связанных с некоторыми комбинаторными оптимизационными задачами. Исследованию условий смежности вершин отдельных политопов, порождаемых подмножествами симметрической группы, посвящены работы [3]-[17]. Указанному классу принадлежат политопы известных классических задач о взвешенном 2- и 3-сочетании, назначении, коммивояжере и различных их модификаций.

Работа выполнена при частичной поддержке фонда INTAS, грант № 03-50-5975.

(C) В. М. ДЕмиденко, 2006 
Наиболее изученным является политоп задачи о назначении. В 1974 г. Балинским и Руссаковым в теоретико-графовой терминологии [3] и, независимо, Падбергом и Рао в терминах подстановок [4] был описан критерий смежности вершин указанного политопа. Ранее в 1955 г. Хэллером [5], [6] были получены условия, определяющие множество вершин этого же политопа, смежных с вершиной, соответствующей тождественной подстановке. В частности, было установлено, что указанное множество вершин порождается всеми циклическими подстановками симметрической группы и только ими (в нашей работе установлено, что критерии смежности Падберга-Рао и условия Хэллера, по существу, идентичны с точностью до некоторого невырожденного линейного преобразования матричного пространства).

Вопросы, связанные с исследованием политопа задачи о коммивояжере, подробно изложены в монографиях [7], [8]. До настоящего времени не известен эффективно проверяемый критерий смежности вершин этого политопа. Известны лишь достаточные условия смежности, полученные в 1969 г. Мурти [9], считавшиеся и необходимыми до выхода в 1976 г. работы Рао [10], в которой установлена ошибочность результата Мурти и предложены новые достаточные условия смежности вершин политопа указанной задачи.

В работах Хаусмана [2], Балаша и Падберга [11] предложены критерии смежности для 0,1-вершин политопа, определяемого целочисленной системой линейных уравнений, и рассмотрены его возможные теоретические и практические применения для описания отдельных классов смежных вершин политопа задачи о коммивояжере. Дополнительные классы смежных вершин с оценками их мощности приведены в [12]. В работе [13] предложена общая схема выделения классов как смежных, так и несмежных вершин, использующая специальные случаи задачи о коммивояжере с заранее известным оптимальным решением. Общий подход для выделения таких случаев предложен в работе [14]. Выход в 1978 г. работы Пападимитриу [15] о NPполноте задачи распознавания несмежности заданной пары вершин указанного политопа делает маловероятной проверку смежности его вершин за полиномиальное время. Попытка характеризации отношения смежности в классе подстановочных политопов впервые предпринята в работах [16], [17].

В данной работе для политопов, порождаемых подмножествами симметрической группы, в терминах подстановок и разрешимости специального вида систем линейных уравнений сформулированы необходимые и достаточные условия смежности их вершин. Предложенные условия позволили построить общую алгоритмическую схему для распознавания смежности вершин подстановочного политопа произвольного вида, оценить ее трудоемкость и указать ситуации, когда реализации этой схемы являются сильно полиномиальными от $n$ для конкретных подмножеств симметрической группы $S_{n}$. В частности, предложенная схема может использоваться для распознавания либо перечисления вершин, смежных с заданной вершиной политопа задачи о коммивояжере, который порождается множеством циклов длины $n$.

2. Предварительные сведения. Пусть $S_{n}$ - симметрическая группа, действующая на множестве чисел $\mathbb{N}_{1, n}=\{1,2, \ldots, n\}$. Подстановка $\sigma \in S_{n}$ трактуется как взаимно однозначное отображение множества $\mathbb{N}_{1, n}$ на себя, которое переводит $i$ в $\sigma(i)$, где $i \in \mathbb{N}_{1, n}$. Носитель подстановки $\sigma$ множество $\operatorname{Tr} \sigma=\left\{i \in \mathbb{N}_{1 n} \mid \sigma(i) \neq i\right\}$ действительно перемещаемых элементов из $\mathbb{N}_{1, n}$ и множество Fix $\sigma=\left\{j \in \mathbb{N}_{1, n} \mid\right.$ $\sigma(j)=j\}$ элементов из $\mathbb{N}_{1, n}$, оставляемых подстановкой $\sigma$ на месте [18; с. 14], обра- 
зуют разбиение $\mathbb{N}_{1, n}$, т.е.

$$
\operatorname{Tr} \sigma \cup \operatorname{Fix} \sigma=\mathbb{N}_{1, n}, \quad \operatorname{Tr} \sigma \cap \operatorname{Fix} \sigma=\varnothing .
$$

Известно [18; с. 17], что произвольная подстановка $\sigma$ допускает разложение в произведение независимых циклов вида

$$
\sigma=\omega_{i_{1}} \circ \omega_{i_{2}} \circ \cdots \circ \omega_{i_{m}}, \quad \text { где } \quad i_{1}, i_{2}, \ldots, i_{m} \in \mathbb{N}_{1, n}, \quad 1 \leqslant m \leqslant n,
$$

которое в дальнейшем называется ииклическим. Напомним, что независимым ииклом $\omega_{i_{\ell}}$ длины $k$ подстановки $\sigma$ называется ее ограничение на подмножестве $\left\{i_{\ell}\right.$, $\left.\sigma\left(i_{\ell}\right), \ldots, \sigma^{k-1}\left(i_{\ell}\right)\right\} \subseteq \mathbb{N}_{1, n}$, где $k$ - минимальный элемент в $\mathbb{N}_{1, n}$ такой, что $\sigma^{k}\left(i_{\ell}\right)=$ $i_{\ell}$, а $\sigma^{k}-k$-я степень подстановки $\sigma$. Так как любая подстановка $\sigma$ полностью определяется ограничением на своем носителе $\operatorname{Tr} \sigma$, который совпадает с объединением попарно не пересекающихся носителей циклов $\omega_{i_{\ell}}$ неединичной длины, то, не ограничивая общности, можно считать, что в разложении (2) циклы длины 1 отсутствуют. Таким образом, если (2) - циклическое разложение $\sigma$, то при $m \geqslant 2$ имеет место

$$
\operatorname{Tr} \sigma=\bigcup_{\ell=1}^{m} \operatorname{Tr} \omega_{i_{\ell}}, \quad\left|\operatorname{Tr} \omega_{i_{\ell}}\right| \geqslant 2, \quad \operatorname{Tr} \omega_{i_{p}} \cap \operatorname{Tr} \omega_{i_{q}}=\varnothing, \text { где } 1 \leqslant p \neq q \leqslant m .
$$

Пусть $\mathbb{R}^{n \times n}$ - пространство квадратных матриц порядка $n$ над полем вещественных чисел $\mathbb{R}$. Любой подстановке $\sigma$ из $S_{n}$ естественным образом ставится в соответствие бинарная матрица $\bar{\sigma}=\left(s_{i, j}\right) \in \mathbb{R}^{n \times n}$ с ненулевыми элементами $s_{i, \sigma(i)}=1$, $i \in \mathbb{N}_{1, n}$, которая называется матрицей подстановки.

Согласно [18; с. 24] любая подстановка $\sigma$ определяет левый сдвиг симметрической группы $S_{n}$, при котором любому подмножеству $H \subseteq S_{n}$ соответствует его левый $\sigma$-сдвиг $\sigma H=\left\{\sigma \circ \eta \in S_{n} \mid \eta \in H\right\}$, где $\sigma \circ \eta$ - произведение подстановок $\sigma, \eta$, действие которого на $S_{n}$ определяется равенствами $\sigma \circ \eta(i)=\sigma(\eta(i)), i \in \mathbb{N}_{1, n}$. С помощью матрицы подстановки $\bar{\sigma}$ естественным образом определяется правый сдвиг пространства $\mathbb{R}^{n \times n}$, при котором любому подмножеству матриц $\mathscr{A} \subseteq \mathbb{R}^{n \times n}$ соответствует его правый $\sigma$-сдвиг $\mathscr{A}^{\sigma}=\left\{A^{\sigma}=A \cdot \bar{\sigma} \mid A \in \mathscr{A}\right\}$. Матрицы вида $A^{\sigma}$ принято называть перенумерованными, так как они получаются перестановкой столбцов исходных матриц с помощью обратной к $\sigma$ подстановки $\sigma^{-1}$, т.е. для любой матрицы $A=\left(a_{i, j}\right)$ ее перенумерованный аналог имеет вид $A^{\sigma}=\left(a_{i, \sigma^{-1}(j)}\right)$.

При доказательстве промежуточных и основных результатов, касающихся свойств подстановочных политопов, существенно используются свойства матриц подстановок и перенумерованных матриц, а именно равенства

$$
\begin{gathered}
\overline{\sigma \circ \rho}=\bar{\rho} \cdot \bar{\sigma}, \quad \overline{\sigma^{-1}}=\bar{\sigma}^{-1}, \\
(A, B)=\left(A^{\sigma}, B^{\sigma}\right), \quad\left(A^{\sigma}\right)^{\rho}=A^{\rho \circ \sigma}, \quad \operatorname{rank} \mathscr{A}=\operatorname{rank} \mathscr{A}^{\sigma},
\end{gathered}
$$

где $\sigma, \rho \in S_{n}, A, B \in \mathbb{R}_{n \times n}, \mathscr{A} \subset \mathbb{R}_{n \times n}$.

Выберем в $S_{n}$ произвольное подмножество $H$. Подстановочным политопом $P_{H}$, порожденным $H$, называется выпуклая оболочка множества матриц подстановок $\bar{H}=\left\{\bar{\eta} \in \mathbb{R}_{n \times n} \mid \eta \in H\right\}$. Выбор подмножества $H \subseteq S_{n}$ определяет вид подстановочного политопа. Например, при $H=S_{n}$ имеем политоп $P_{S_{n}}$ классической задачи 
о назначениях. Если $H$ совпадает с множеством $T_{n}$ всех циклов длины $n$, то $P_{T_{n}}-$ политоп известной задачи о коммивояжере. Известно, что при любом $H \subseteq S_{n}$ политоп $P_{H}$ является вырожденным в $\mathbb{R}^{n \times n}$, т.е. принадлежит минимальному аффинному пространству aff $P_{H}$, размерность которого не превосходит $\operatorname{dim}\left(\right.$ aff $\left.P_{S_{n}}\right)=(n-1)^{2}$. Известно, что множество вершин политопа $P_{H}$ совпадает с $\bar{H}$. Далее, две вершины политопа $P_{H}$ называются смежными, если они принадлежат одному и тому же ребру (грани политопа $P_{H}$ размера 1$)$.

При выборе основных понятий и обозначений, связанных с исследованием отношения смежности вершин подстановочных политопов, мы придерживаемся терминологии монографий [19] и [20]. При этом наряду с выпукло и аффинно независимыми множествами пространства $\mathbb{R}^{n \times n}[19 ;$ с. 14] рассматриваются конически независимые. Любая матрица каждого такого множества не может быть выражена в виде неотрицательной линейной комбинации его остальных матриц. В дальнейшем такие линейные комбинации матриц называются коническими.

Введем в рассмотрение множество $\mathscr{E}=\left\{E_{i, j} \in \mathbb{R}^{n \times n} \mid i, j \in \mathbb{N}_{1, n}\right\}$ бинарных матриц, в каждой из которых единственный ненулевой элемент стоит на пересечении $i$-й строки и $j$-го столбца. Из (1) и определения матриц подстановок непосредственно следуют равенства вида

$$
\bar{\sigma}=\sum_{i \in \operatorname{Tr} \sigma} E_{i, \sigma(i)}+\sum_{j \in \operatorname{Fix} \sigma} E_{j, j}, \quad \bar{\sigma}-\bar{\varepsilon}=\sum_{i \in \operatorname{Tr} \sigma} E_{i, \sigma(i)}-\sum_{i \in \operatorname{Tr} \sigma} E_{i, i},
$$

которые задают разложение по базису $\mathscr{E}$ матриц подстановок $\bar{\sigma}$ и $\bar{\sigma}-\bar{\varepsilon}$, где $\sigma-$ произвольная, а $\varepsilon$ - тождественная подстановки из $S_{n}$.

В данной работе формулируется критерий (не)смежности вершин политопов вида $P_{\eta^{-1} H}$, где $H \subseteq S_{n}$ и $\eta \in H$, которые образуют некоторый подкласс в классе всех подстановочных политопов. Однако наличие взаимно однозначного соответствия между гранями политопов $P_{\eta^{-1} H}$ и $P_{H}$ позволяет расширить предлагаемый критерий на весь класс подстановочных политопов. Свойства этого соответствия $\left(\eta^{-1}\right.$ сдвига матричного пространства), касающиеся подстановочных политопов, описываются в п. 3.

3. Грани политопов $P_{\eta^{-1} H}$ и $P_{H}$ и смежность их вершин. При изучении отношения смежности вершин подстановочных политопов будем рассматривать не сам политоп $P_{H}$, а его правый $\eta^{-1}$-сдвиг $P_{\eta^{-1} H}=\left(P_{H}\right)^{\eta^{-1}}$, где $\eta$ - произвольно выбранная в $H$ подстановка. Покажем, что полученный политоп $P_{\eta^{-1} H}$ полностью идентичен $P_{H}$ в смысле строения граней.

Пусть $\mathscr{A} \subset \mathbb{R}^{n \times n}$ и $\sigma \in S_{n}$. Введем в рассмотрение систему линейных неравенств

$$
\operatorname{sli} \mathscr{A}=\left\{(A, X) \leqslant b_{A} \mid A \in \mathscr{A}, b_{A} \in \mathbb{R}\right\}
$$

допускающую включение неявных либо явных равенств, где $X=\left(x_{i, j}\right)$ - матрица переменных $x_{i, j}, i, j \in \mathbb{N}_{1, n}$, a

$$
(A, X)=\sum_{i=1}^{n} \sum_{j=1}^{n} a_{i, j} x_{i, j}
$$


- скалярное произведение матриц $A$ и $X$. Систему линейных уравнений, которая получается из sli $\mathscr{A}$ заменой всех знаков ' $\leqslant$ ' на '=', обозначим sle $\mathscr{A}$. Перенумерованной системой sli $\mathscr{A}$ посредством $\sigma$ назовем систему

$$
\text { sli } \mathscr{A}^{\sigma}=\left\{\left(A^{\sigma}, X\right) \leqslant b_{A} \mid A \in \mathscr{A}, b_{A} \in \mathbb{R}\right\} .
$$

Связь между гранями политопов $P_{H}$ и $P_{\eta^{-1}}$ устанавливает следующее

ПреДЛОЖениЕ 1. Семейство подмножеств $\mathscr{F}=\left\{F_{\ell} \subset P_{H} \mid \ell \in J\right\}$, әде Jконечное множество индексов, является семейством граней политопа $P_{H}$ тогда и только тогда, когда $\mathscr{F}_{\eta^{-1}}=\left\{F_{\ell}^{\eta^{-1}} \subset P_{\eta^{-1} H} \mid \ell \in J\right\}-$ семейство граней политоna $P_{\eta^{-1} H}$.

ДокАЗАТЕЛЬСтво. Предложение 1 является прямым следствием следующего утверждения: подмножество $F$ политопа $P_{H}$ является его $k$-мерной гранью тогда и только тогда, когда $F^{\eta^{-1}}-k$-мерная грань политопа $P_{\eta^{-1} H}$.

Докажем вначале необходимость условий сформулированного утверждения. Согласно [20; с. 136] любой конечно порожденный политоп совпадает с множеством решений некоторой системы линейных неравенств, допускающей включение неявных либо явных равенств. Пусть, для определенности, политоп $P_{H}-$ множество решений системы линейных неравенств

$$
\operatorname{sli} \mathscr{A}=\left\{(A, X) \leqslant b_{A} \mid A \in \mathscr{A} \subset \mathbb{R}^{n \times n}, b_{A} \in \mathbb{R}\right\} .
$$

Используя равенства (4) и (5), нетрудно проверить, что $P_{\eta^{-1} H}=\left(P_{H}\right)^{\eta^{-1}}$ - множество решений перенумерованной системы вида

$$
\operatorname{sli} \mathscr{A}^{\eta^{-1}}=\left\{\left(A^{\eta^{-1}}, X\right) \leqslant b_{A} \mid A \in \mathscr{A}, b_{A} \in \mathbb{R}\right\} .
$$

Далее, согласно [19; с. 33] непустое подмножество $F$ политопа $P_{H}$ является его $k$ мерной гранью, если оно совпадает с множеством решений системы уравнений sle $\mathscr{A}_{1}$, определяемой некоторой подсистемой sli $\mathscr{A}_{1} \subset$ sli $\mathscr{A}$ ранга $n^{2}-k$. Таким образом, если $k$-мерная грань $F$ - решение системы уравнений sle $\mathscr{A}_{1}$ ранга $n^{2}-k$, то, используя равенства (4) и (5), нетрудно убедиться в том, что непустое подмножество $F^{\eta^{-1}}$ политопа $P_{\eta^{-1} H}$ является решением перенумерованной системы уравнений sle $\mathscr{A}_{1}^{\eta^{-1}}$. Эта система в силу последнего равенства (5) имеет ранг $n^{2}-k$ и, очевидно, определяется подсистемой sli $\mathscr{A}_{1}^{\eta^{-1}}$ системы sli $\mathscr{A}^{\eta^{-1}}$, множество решений которой совпадает с $P_{\eta^{-1} H}$. Таким образом, $F^{\eta^{-1}}$ является $k$-мерной гранью политопа $P_{\eta^{-1}}$.

Убедимся теперь в справедливости обратного утверждения. Так как имеет место равенство $P_{\eta^{-1} H}=\left(P_{H}\right)^{\eta^{-1}}$, то любая $k$-мерная грань политопа $P_{\eta^{-1} H}$ имеет вид $F^{\eta^{-1}}$, где $F$ - некоторое непустое подмножество политопа $P_{H}$. Далее, так как $P_{\eta^{-1} H}$ - решение системы sli $\mathscr{A}^{-1}$, то грань $F^{\eta^{-1}}$ является решением системы уравнений sle $\mathscr{A}_{1}^{\eta^{-1}}$ ранга $n^{2}-k$, где $\mathscr{A}_{1} \subseteq \mathscr{A}$. В силу первых двух равенств (5) перенумерованное подмножество $\left(F^{\eta^{-1}}\right)^{\eta}=F$ совпадает с множеством решений системы уравнений

$$
\operatorname{sle}\left(\mathscr{A}_{1}^{\eta^{-1}}\right)^{\eta}=\operatorname{sle} \mathscr{A}_{1}
$$

которая определяется подсистемой sli $\mathscr{A}_{1}$ системы sli $\mathscr{A}$ и имеет в силу последнего равенства (5) ранг $n^{2}-k$. Так как sli $\mathscr{A}$ определяет политоп $P_{H}$, то $F$ - его $k$-мерная грань. Таким образом, предложение 1 доказано. 
Ранее отмечалось, что $\bar{H}$ - множество вершин подстановочного политопа $P_{H}$. Следовательно, множество вершин политопа $P_{\eta^{-1} H}$ в силу предложения 1 совпадает с множеством перенумерованных матриц подстановок $\bar{H}^{\eta^{-1}}$. Ниже формулируются утверждения, устанавливающие связь между смежными вершинами политопов $P_{H}$ и $P_{\eta^{-1} H}$.

Теорема 1. Вериины $\bar{\eta} u \bar{v}$ политопа $P_{H}$ являются смежными тогда и толъко тогда, когда смежны вериины $\bar{\varepsilon} u \bar{v} \cdot \bar{\eta}^{-1}$ политопа $P_{\eta^{-1} H}$.

ДокАЗАТЕЛЬСТво. По определению две вершины политопа смежны, если они принадлежат одному и тому же ребру, т.е. грани размера 1 . В силу предложения 1 каждой грани $F$ размера 1 политопа $P_{H}$ соответствует грань $F^{\eta-1}$ той же размерности политопа $P_{\eta^{-1}}$. Следовательно, чтобы доказать теорему 1 , достаточно убедиться в справедливости двух утверждений: если $\bar{\eta}, \bar{v} \in F$, то $\bar{\varepsilon}, \bar{v} \cdot \bar{\eta}^{-1} \in F^{\eta^{-1}}$, и если $\bar{\varepsilon}, \bar{v} \cdot \bar{\eta}^{-1} \in F^{\eta^{-1}}$, то $\bar{\eta}, \bar{v} \in F$.

Убедимся в справедливости первого утверждения. Пусть система неравенств

$$
\text { sli } \mathscr{A}=\left\{(A, X) \leqslant b_{A} \mid A \in \mathscr{A} \subset \mathbb{R}^{n \times n}, b_{A} \in \mathbb{R}\right\}
$$

определяет политоп $P_{H}$. Согласно [19; с. 33] 1-мерная грань $F$ политопа $P_{H}$ является множеством решений системы

$$
\text { sle } \mathscr{A}_{1}=\left\{(A, X)=b_{A} \mid A \in \mathscr{A}_{1} \subset \mathscr{A}, b_{A} \in \mathbb{R}\right\}
$$

ранга $n^{2}-1$, которая порождается некоторой подсистемой sli $\mathscr{A}_{1} \subset$ sli $\mathscr{A}$. Из предложения 1 и двух первых равенств (5) следует, что 1 -мерная грань $F^{\eta^{-1}} \subset P_{\eta^{-1} H}$ определяется системой

$$
\text { sle } \mathscr{A}_{1}^{\eta^{-1}}=\left\{\left(A^{\eta^{-1}}, X\right)=b_{A} \mid A \in \mathscr{A}_{1}, b_{A} \in \mathbb{R}\right\},
$$

ранг которой в силу третьего равенства (5) равен $n^{2}-1$.

Пусть $\bar{\eta}, \bar{v} \in F$, тогда для всех $A \in \mathscr{A}_{1}$ должны выполняться равенства $(A, \bar{\eta})=b_{A}$, $(A, \bar{v})=b_{A}$, из которых и $(4),(5)$ вытекают цепочки равенств

$$
\begin{aligned}
& b_{A}=(A, \bar{\eta})=\left(A^{\eta^{-1}}, \bar{\eta}^{\eta^{-1}}\right)=\left(A^{\eta^{-1}}, \bar{\eta} \cdot \bar{\eta}^{-1}\right)=\left(A^{\eta^{-1}}, \overline{\eta^{-1} \circ \eta}\right)=\left(A^{\eta^{-1}}, \bar{\varepsilon}\right), \\
& b_{A}=(A, \bar{v})=\left(A^{\eta^{-1}}, \bar{v}^{\eta^{-1}}\right)=\left(A^{\eta^{-1}}, \bar{v} \cdot \overline{\eta^{-1}}\right)=\left(A^{\eta^{-1}}, \bar{v} \cdot \bar{\eta}^{-1}\right),
\end{aligned}
$$

имеющие место для всех $A$ из $\mathscr{A}_{1}$. Следовательно, $\bar{\varepsilon}$ и $\bar{v} \cdot \bar{\eta}^{-1}$ - решения системы sle $\mathscr{A}_{1}^{\eta^{-1}}$ ранга $n^{2}-1$, что влечет $\bar{\varepsilon}, \bar{v} \cdot \bar{\eta}^{-1} \in F^{\eta^{-1}}$. Таким образом, доказано, что $\bar{\varepsilon}$ и $\bar{v} \cdot \bar{\eta}^{-1}$ - смежные вершины политопа $P_{\eta^{-1} H}$.

Пусть теперь $\bar{\varepsilon}$ и $\bar{v} \cdot \bar{\eta}^{-1}$ - смежные вершины политопа $P_{\eta^{-1} H}$ и они принадлежат $F^{\eta^{-1}}$. Тогда они являются решениями системы уравнений sle $\mathscr{A}_{1}^{\eta^{-1}}$ и для всех $A \in \mathscr{A}_{1}$ имеет место

$$
\left(A^{\eta^{-1}}, \bar{\varepsilon}\right)=\left(A^{\eta^{-1}}, \bar{v} \cdot \bar{\eta}^{-1}\right)=b_{A} .
$$

Следовательно, в силу (4) и первых двух равенств (5) должны выполняться равенства

$$
\begin{aligned}
& b_{A}=\left(A^{\eta^{-1}}, \bar{\varepsilon}\right)=\left(\left(A^{\eta^{-1}}\right)^{\eta}, \bar{\varepsilon}^{\eta}\right)=\left(A^{\eta \circ \eta^{-1}}, \overline{\varepsilon \circ \eta}\right)=(A, \bar{\eta}), \\
& b_{A}=\left(A^{\eta^{-1}}, \bar{v} \cdot \bar{\eta}^{-1}\right)=\left(\left(A^{\eta^{-1}}\right)^{\eta},\left(\bar{v} \cdot \bar{\eta}^{-1}\right)^{\eta}\right)=\left(A^{\eta \circ \eta^{-1}}, \overline{\eta \circ \eta^{-1} \circ v}\right)=(A, \bar{v})
\end{aligned}
$$


для всех $A$ из $\mathscr{A}_{1}$. Таким образом, матрицы $\bar{\eta}$ и $\bar{v}$ - решения системы sle $\mathscr{A}_{1}$, т.е. являются смежными вершинами политопа $P_{H}$. Теорема 1 доказана.

4. Необходимые условия несмежности вершин политопа $P_{\eta^{-1} H} \cdot$ При доказательстве необходимых и достаточных условий несмежности вершин подстановочных политопов, представленных в последующих пунктах, существенно используется критерий смежности [16], сформулированный в терминах подстановок и специального вида конических разложений соответствующих им матриц.

Зафиксируем в множестве вершин $\bar{H}$ политопа $P_{H}$ любую вершину $\bar{\eta}_{0}$ и обозначим $\bar{H}_{\eta_{0}}$ подмножество смежных с $\bar{\eta}_{0}$ вершин. Очевидно, $\bar{H}_{\eta_{0}} \subseteq \bar{H}$ определяет в $H$ соответствующее подмножество подстановок $H_{\eta_{0}}$. Ниже приводятся необходимые и достаточные условия принадлежности подстановок подмножеству $H \backslash H_{\eta_{0}}$. Эти условия, по существу, задают в терминах подстановок критерий несмежности вершин политопа $P_{H}$ с вершиной $\bar{\eta}_{0}$.

Теорема 2. При заданных $H \subseteq S_{n} u \eta_{0}, \eta \in H$, где $|H| \geqslant 4$, подстановка $\eta$ принадлежит подмножеству $H \backslash H_{\eta_{0}}$ тогда и толъко тогда, когда найдется подмножество $\Upsilon \subseteq H \backslash\left\{\eta_{0}, \eta\right\}$ мощности $|\Upsilon| \geqslant 2$ и набор чисел $0<\lambda_{v} \in \mathbb{R}$, где $v \in \Upsilon$, такие, что для произвольной матрицы $X \in \mathbb{R}^{n \times n}$ выполняется равенство

$$
\left(\bar{\eta}-\bar{\eta}_{0}, X\right)=\sum_{v \in \Upsilon} \lambda_{v}\left(\bar{v}-\bar{\eta}_{0}, X\right)
$$

Из теорем 1, 2 вытекает критерий несмежности вершин политопа $P_{\eta^{-1} H}$ с вершиной $\bar{\varepsilon}$, соответствующей тождественной подстановке.

СлеДСТВие 1. Пусть $H \subseteq S_{n}, \eta \in H u v_{0} \in \eta^{-1} H$. Вериины $\bar{v}_{0} u \bar{\varepsilon}$ полито-

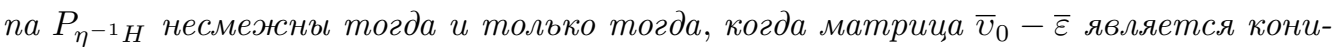
ческой комбинацией множества матрии $\{\bar{v}-\bar{\varepsilon} \mid v \in \Upsilon\}$, где $\subseteq \eta^{-1} H \backslash\left\{v_{0}, \varepsilon\right\}$ u $|\Upsilon| \geqslant 2$.

Условия следствия 1 предполагают выполнение матричного равенства

$$
\bar{v}_{0}-\bar{\varepsilon}=\sum_{v \in \Upsilon} \lambda_{v}(\bar{v}-\bar{\varepsilon}), \quad \text { где } \quad 0<\lambda_{v} \in \mathbb{R}, \quad \Upsilon \subseteq \eta^{-1} H \backslash\left\{v_{0}, \varepsilon\right\} .
$$

Ниже рассматриваются свойства подстановок из $\Upsilon \subseteq \eta^{-1} H \backslash\left\{v_{0}, \varepsilon\right\}$, входящих в (7). Эти свойства определяют в силу теоремы 1 и следствия 1 необходимые условия несмежности вершин подстановочных политопов и представляют определенный теоретический и практический интерес.

ПРЕДЛОЖЕНИЕ 2. Пусть $H \subseteq S_{n} u \eta \in H$ таковы, что для подстановки $v_{0} \in$ $\eta^{-1} H$ и подмножества $\Upsilon \subseteq \eta^{-1} H \backslash\left\{v_{0}, \varepsilon\right\}$, где $|\Upsilon| \geqslant 2$, имеет место матричное равенство (7). Тогда для любой подстановки $v \in \Upsilon$ имеет место

$$
\operatorname{Tr} v \subset \operatorname{Tr} v_{0}, \quad \bigcup_{v \in \Upsilon} \operatorname{Tr} v=\operatorname{Tr} v_{0} \quad u \quad v(i)=v_{0}(i), \text { где } i \in \operatorname{Tr} v
$$

ДокАЗАтельство. В начале убедимся в том, что равенства $v(i)=v_{0}(i)$, где $i \in \operatorname{Tr} v$, выполняются для любой подстановки $v \in \Upsilon$. Для этого достаточно доказать невозможность противоположного утверждения, т.е. отсутствие в $\Upsilon$ такой 
подстановки $v^{\prime}$, что $v^{\prime}(\ell) \neq v_{0}(\ell)$ для некоторого элемента $\ell$ из $\operatorname{Tr} v^{\prime}$. Предположим наличие в $\Upsilon$ подстановки $v^{\prime}$ и выделим подмножество $\Upsilon^{\prime}$ всех таких подстановок $v \in \Upsilon$, что $\ell \in \operatorname{Tr} v$ и $v(\ell)=v^{\prime}(\ell)$. Очевидно, $\Upsilon^{\prime} \neq \varnothing$ ввиду того, что $v^{\prime} \in \Upsilon^{\prime}$. Далее, так как для матриц, соответствующих подстановкам $v_{0}, \varepsilon, v \in \Upsilon$, справедливо равенство (7), то скалярное произведение обеих его частей на матрицу $E_{\ell, v^{\prime}(\ell)}$ должно дать верное числовое равенство.

Скалярно умножая левую часть $(7)$ на $E_{\ell, v^{\prime}(\ell)}$ и используя первое равенство (6), получим следующую цепочку равенств:

$$
\begin{aligned}
\left(\bar{v}_{0}-\bar{\varepsilon}, E_{\ell, v^{\prime}(\ell)}\right) & =\left(\bar{v}_{0}, E_{\ell, v^{\prime}(\ell)}\right)-\left(\bar{\varepsilon}, E_{\ell, v^{\prime}(\ell)}\right) \\
& =\sum_{i \in \mathbb{N}_{1, n}}\left(E_{i, v_{0}(i)}, E_{\ell, v^{\prime}(\ell)}\right)-\sum_{i \in \mathbb{N}_{1, n}}\left(E_{i, i}, E_{\ell, v^{\prime}(\ell)}\right) \\
& =\left(E_{\ell, v_{0}(\ell)}, E_{\ell, v^{\prime}(\ell)}\right)-\left(E_{\ell, \ell}, E_{\ell, v^{\prime}(\ell)}\right)=0 .
\end{aligned}
$$

Действительно, при $i \neq \ell$ имеем $\left(E_{i, v_{0}(i)}, E_{\ell, v^{\prime}(\ell)}\right)=0$ и $\left(E_{i, i}, E_{\ell, v^{\prime}(\ell)}\right)=0$. Далее равенство $\left(E_{\ell, v_{0}(\ell)}, E_{\ell, v^{\prime}(\ell)}\right)=0$ выполняется в силу $v_{0}(\ell) \neq v^{\prime}(\ell)$, a $\left(E_{\ell, \ell}, E_{\ell, v^{\prime}(\ell)}\right)=0$, так как имеет место $\ell \neq v^{\prime}(\ell)$ ввиду того, что $\ell \in \operatorname{Tr} v$.

Скалярное умножение правой части матричного равенства $(7)$ на матрицу $E_{\ell, v^{\prime}(\ell)}$ с учетом первого равенства (6) приводит к цепочке равенств

$$
\begin{aligned}
& \left(\sum_{v \in \Upsilon} \lambda_{v}(\bar{v}-\bar{\varepsilon}), E_{\ell, v^{\prime}(\ell)}\right)=\sum_{v \in \Upsilon} \lambda_{v}\left(\bar{v}-\bar{\varepsilon}, E_{\ell, v^{\prime}(\ell)}\right) \\
& \quad=\sum_{v \in \Upsilon} \lambda_{v}\left(\sum_{i \in \mathbb{N}_{1, n}}\left(E_{i, v(i)}, E_{\ell, v^{\prime}(\ell)}\right)\right)-\sum_{v \in \Upsilon} \lambda_{v}\left(\sum_{i \in \mathbb{N}_{1, n}}\left(E_{i, i}, E_{\ell, v^{\prime}(\ell)}\right)\right) \\
& \quad=\sum_{v \in \Upsilon} \lambda_{v}\left(E_{\ell, v(\ell)}, E_{\ell, v^{\prime}(\ell)}\right),
\end{aligned}
$$

так как $\left(E_{i, v(\ell)}, E_{\ell, v^{\prime}(\ell)}\right)=0$ для всех $i \neq \ell \in \mathbb{N}_{1, n}$, а $\left(E_{i, i}, E_{\ell, v^{\prime}(\ell)}\right)=0$ для всех $i \in \mathbb{N}_{1, n}$, так как ввиду того, что $\ell \in \operatorname{Tr} v^{\prime}$, имеет место $\ell \neq v^{\prime}(\ell)$.

Далее из $\Upsilon=\Upsilon^{\prime} \cup\left(\Upsilon \backslash \Upsilon^{\prime}\right), \Upsilon^{\prime} \cap\left(\Upsilon \backslash \Upsilon^{\prime}\right)=\varnothing,\left(E_{\ell, v(\ell)}, E_{\ell, v^{\prime}(\ell)}\right)=1$ при $v \in \Upsilon^{\prime}$ и $\left(E_{\ell, v(\ell)}, E_{\ell, v^{\prime}(\ell)}\right)=0$ при $v \in \Upsilon \backslash \Upsilon^{\prime}$ следует справедливость равенств

$$
\begin{aligned}
\sum_{v \in \Upsilon} \lambda_{v}\left(E_{\ell, v(\ell)}, E_{\ell, v^{\prime}(\ell)}\right) & =\sum_{v \in \Upsilon^{\prime}} \lambda_{v}\left(E_{\ell, v(\ell)}, E_{\ell, v^{\prime}(\ell)}\right)+\sum_{v \in \Upsilon \backslash \Upsilon^{\prime}} \lambda_{v}\left(E_{\ell, v(\ell)}, E_{\ell, v^{\prime}(\ell)}\right) \\
& =\sum_{v \in \Upsilon^{\prime}} \lambda_{v}\left(E_{\ell, v(\ell)}, E_{\ell, v^{\prime}(\ell)}\right)=\sum_{v \in \Upsilon^{\prime}} \lambda_{v}>0 .
\end{aligned}
$$

Сравнение вычисленных скалярных произведений дает противоречие

$$
0=\left(\bar{v}_{0}-\bar{\varepsilon}, E_{\ell, v^{\prime}(\ell)}\right)=\left(\sum_{v \in \Upsilon} \lambda_{v}(\bar{v}-\bar{\varepsilon}), E_{\ell, v^{\prime}(\ell)}\right)=\sum_{v \in \Upsilon^{\prime}} \lambda_{v}>0,
$$

что доказывает справедливость равенств $v(i)=v_{0}(i)$, где $i \in \operatorname{Tr} v, v \in \Upsilon$.

Убедимся теперь в справедливости строгого включения $\operatorname{Tr} v \subset \operatorname{Tr} v_{0}$ для любой подстановки $v \in \Upsilon$. Очевидно, $\operatorname{Tr} v \neq \operatorname{Tr} v_{0}$, так как в противном случае из доказанных выше равенств и $\operatorname{Tr} v=\operatorname{Tr} v_{0}$ следует $v=v_{0}$, что противоречит выбору $\Upsilon$. 
Если $\operatorname{Tr} v_{0} \subset \operatorname{Tr} v$, то в $\operatorname{Tr} v$ должен существовать такой элемент $\ell$, что $\ell \notin \operatorname{Tr} v_{0}$, т.е. в силу (1) $\ell \in \mathbb{N}_{1, n} \backslash \operatorname{Tr} v_{0}=\operatorname{Fix} v_{0}$, откуда следует $v_{0}(\ell)=\ell \neq v(\ell)$. Полученное противоречие с выше установленным свойством подстановок $v$ из $\Upsilon\left(v(i)=v_{0}(i)\right.$, где $i \in \operatorname{Tr} v$ и $v \in \Upsilon$ ) доказывает выполнение требуемых включений $\operatorname{Tr} v \subset \operatorname{Tr} v_{0}$ для всех $v \in \Upsilon$. Следствием последних является включение

$$
\bigcup_{v \in \Upsilon} \operatorname{Tr} v \subseteq \operatorname{Tr} v_{0}
$$

Таким образом, для завершения доказательства предложения 2 осталось убедиться в справедливости обратного включения

$$
\operatorname{Tr} v_{0} \subseteq \bigcup_{v \in \Upsilon} \operatorname{Tr} v
$$

из которого и предыдущего будет следовать требуемое равенство

$$
\operatorname{Tr} v_{0}=\bigcup_{v \in \Upsilon} \operatorname{Tr} v
$$

Предположим, что

$$
\operatorname{Tr} v_{0} \nsubseteq \bigcup_{v \in \Upsilon} \operatorname{Tr} v
$$

Тогда найдется элемент $\ell \in \operatorname{Tr} v_{0}$ такой, что $\ell \notin \operatorname{Tr} v$ для всех $v \in \Upsilon$ и, следовательно, в силу (1) для всех $v \in \Upsilon$ имеет место $\ell \in \operatorname{Fix} v$, что равносильно $v(\ell)=\ell$. Покажем, что при сделанных предположениях скалярное умножение левой и правой части равенства (7) на $E_{\ell, v_{0}(\ell)}$ не равны. Так как $\left(E_{i, v_{0}(i)}, E_{\ell, v_{0}(\ell)}\right)=0$ для всех $i \neq \ell \in \mathbb{N}_{1, n}$, а $\left(E_{\ell, \ell}, E_{\ell, v_{0}(\ell)}\right)=0$, ввиду $\ell \in \operatorname{Tr} v_{0}$ и $\left(E_{\ell, v_{0}(\ell)}, E_{\ell, v_{0}(\ell)}\right)=1$, то скалярное умножение левой части $(7)$ на $E_{\ell, v_{0}}(\ell)$ дает цепочку равенств

$$
\left(\bar{v}_{0}-\bar{\varepsilon}, E_{\ell, v_{0}(\ell)}\right)=\sum_{i \in \mathbb{N}_{1, n}}\left(E_{i, v_{0}(i)}, E_{\ell, v_{0}(\ell)}\right)-\sum_{i \in \mathbb{N}_{1, n}}\left(E_{i, i}, E_{\ell, v_{0}(\ell)}\right)=\left(E_{\ell, v_{0}(\ell)}, E_{\ell, v_{0}(\ell)}\right)=1 .
$$

Умножая скалярно правую часть равенства $(7)$ на $E_{\ell, v_{0}(\ell)}$, получим

$$
\begin{aligned}
\left(\sum_{v \in \Upsilon} \lambda_{v}(\bar{v}-\bar{\varepsilon}), E_{\ell, v_{0}(\ell)}\right) & =\sum_{v \in \Upsilon} \lambda_{v}\left(\sum_{i \in \mathbb{N}_{1, n}}\left(E_{i, v(i)}-E_{i, i}, E_{\ell, v_{0}(\ell)}\right)\right) \\
& =\sum_{v \in \Upsilon} \lambda_{v}\left(\left(E_{\ell, v(\ell)}, E_{\ell, v_{0}(\ell)}\right)-\left(E_{\ell, \ell}, E_{\ell, v_{0}(\ell)}\right)\right)=0,
\end{aligned}
$$

так как $\left(E_{i, v(i)}, E_{\ell, v_{0}(\ell)}\right)=\left(E_{i, i}, E_{\ell, v_{0}(\ell)}\right)=0$ при $i \neq \ell \in \mathbb{N}_{1, n},\left(E_{\ell, v(\ell)}, E_{\ell, v_{0}(\ell)}\right)=$ $\left(E_{\ell, \ell}, E_{\ell, v_{0}(\ell)}\right)=0$, так как для $\ell$, в силу определения, должны выполняться соотношения $v(\ell)=\ell$ и $v_{0}(\ell) \neq \ell$. Полученное противоречие доказывает справедливость включения

$$
\operatorname{Tr} v_{0} \subseteq \bigcup_{v \in \Upsilon} \operatorname{Tr} v
$$

Предложение 2 доказано. 
ПреДЛОЖЕНИЕ 3. Пусть $H \subseteq S_{n}, \eta \in H$ u для подстановки $v_{0} \in \eta^{-1} H$ с множеством независимых ииклов $\Omega_{v_{0}}=\left\{\omega_{i} \mid i \in \mathbb{N}_{1, m}\right\}$, где $2 \leqslant m \leqslant\lfloor n / 2\rfloor$, существует такое подмножество $\Upsilon \subseteq \eta^{-1} H \backslash\left\{v_{0}, \varepsilon\right\}$ мощности $|\Upsilon| \geqslant 2$, что выполняется матричное равенство (7). Тогда для любой подстановки $v \in \Upsilon$ существует такое подмножество $J_{v} \subset \mathbb{N}_{1, m}$, что

$$
v=\prod_{j \in J_{v}} \omega_{j}
$$

ДокАЗАТЕЛЬСТвО. Чтобы убедиться в справедливости предложения 3, достаточно показать невозможность противоположного вывода при заданных условиях. Предположим существование в $\Upsilon$ такой подстановки $v^{\prime}$, что

$$
v^{\prime} \neq \prod_{j \in J} \omega_{j}
$$

для любого подмножества $J \subset \mathbb{N}_{1, m}$, где $\omega_{j} \in \Omega_{v_{0}}$. В этом случае среди независимых циклов подстановки $v^{\prime}$ должен найтись такой цикл $\omega^{\prime}$, что $\omega^{\prime} \neq \omega_{i}$ для всех $i \in$ $\mathbb{N}_{1, m}$ (в противном случае все независимые циклы подстановки $v^{\prime}$ принадлежат $\Omega_{v_{0}}$ ). Из приведенных соотношений следует существование для каждого цикла $\omega_{i}$ из $\Omega_{v_{0}}$ элемента $\ell_{i} \in \operatorname{Tr} \omega^{\prime}$ такого, что $\omega^{\prime}\left(\ell_{i}\right) \neq \omega_{i}\left(\ell_{i}\right)$ (в противном случае $\omega^{\prime}=\omega_{i}$ ). Далее, в силу предложения 2 и имеющих место включений $v^{\prime} \in \Upsilon, \operatorname{Tr} \omega^{\prime} \subseteq \operatorname{Tr} v^{\prime}$ и $\operatorname{Tr} \omega_{i} \subseteq$ $\operatorname{Tr} v_{0}$, должны выполняться равенства

$$
\omega^{\prime}\left(\ell_{i}\right)=v^{\prime}\left(\ell_{i}\right)=v_{0}\left(\ell_{i}\right) \quad \text { и } \quad \omega_{i}\left(\ell_{i}\right)=v_{0}\left(\ell_{i}\right),
$$

из которых и доказанного выше соотношения $\omega^{\prime}\left(\ell_{i}\right) \neq \omega_{i}\left(\ell_{i}\right)$ следует цепочка

$$
v_{0}\left(\ell_{i}\right)=\omega^{\prime}\left(\ell_{i}\right) \neq \omega_{i}\left(\ell_{i}\right)=v_{0}\left(\ell_{i}\right)
$$

Полученное противоречие доказывает справедливость предложения 3.

5. Достаточные условия несмежности вершин политопа $P_{\eta^{-1} H}$. При доказательстве результатов этого пункта понадобятся следующие утверждения.

Лемма 1. Пусть $\sigma, \rho \in S_{n} u \operatorname{Tr} \sigma \cap \operatorname{Tr} \rho=\varnothing$. Тогда справедливъь равенства $(\bar{\sigma}-\bar{\varepsilon}, \bar{\rho}-\bar{\varepsilon})=0 u(\bar{\sigma}-\bar{\varepsilon}) \cdot(\bar{\rho}-\bar{\varepsilon})=O$, где $O-$ нулевая матрииа.

ДокАЗАТЕЛЬСтво. Непосредственное вычисление скалярного произведения с учетом второго равенства (6) приводит к цепочке равенств

$$
\begin{aligned}
& (\bar{\sigma}-\bar{\varepsilon}, \bar{\rho}-\bar{\varepsilon})=\left(\sum_{i \in \operatorname{Tr} \sigma} E_{i, \sigma(i)}-\sum_{i \in \operatorname{Tr} \sigma} E_{i, i}, \sum_{j \in \operatorname{Tr} \rho} E_{j, \rho(j)}-\sum_{j \in \operatorname{Tr} \rho} E_{j, j}\right) \\
& =\sum_{i \in \operatorname{Tr} \sigma} \sum_{j \in \operatorname{Tr} \rho}\left(\left(E_{i, \sigma(i)}, E_{j, \rho(j)}\right)-\left(E_{i, \sigma(i)}, E_{j, j}\right)-\left(E_{i, i}, E_{j, \rho(j)}\right)+\left(E_{i, i}, E_{j, j}\right)\right)=0,
\end{aligned}
$$

так как $i \neq j$ для всех $i \in \operatorname{Tr} \sigma$ и $j \in \operatorname{Tr} \rho$ ввиду $\operatorname{Tr} \sigma \cap \operatorname{Tr} \rho=\varnothing$, что влечет равенство нулю всех слагаемых указанной выше двойной суммы. 
Докажем справедливость матричного равенства. Из свойств операции умножения матриц и второго равенства (6) следует цепочка равенств

$$
\begin{aligned}
(\bar{\sigma}-\bar{\varepsilon}) \cdot(\bar{\rho}-\bar{\varepsilon})= & \left(\sum_{i \in \operatorname{Tr} \sigma} E_{i, \sigma(i)}-\sum_{i \in \operatorname{Tr} \sigma} E_{i, i}\right) \cdot\left(\sum_{j \in \operatorname{Tr} \rho} E_{j, \rho(j)}-\sum_{j \in \operatorname{Tr} \rho} E_{j, j}\right) \\
= & \sum_{i \in \operatorname{Tr} \sigma} \sum_{j \in \operatorname{Tr} \rho} E_{i, \sigma(i)} \cdot E_{j, \rho(j)}-\sum_{i \in \operatorname{Tr} \sigma} \sum_{j \in \operatorname{Tr} \rho} E_{i, \sigma(i)} \cdot E_{j, j} \\
& -\sum_{i \in \operatorname{Tr} \sigma} \sum_{j \in \operatorname{Tr} \rho} E_{i, i} \cdot E_{j, \rho(j)}+\sum_{i \in \operatorname{Tr} \sigma} \sum_{j \in \operatorname{Tr} \rho} E_{i, i} \cdot E_{j, j} .
\end{aligned}
$$

Докажем совпадение с нулевой матрицей $O$ всех слагаемых полученных двойных сумм. Проверка показывает, что соотношения $E_{p, q} \cdot E_{r, s} \neq O$ выполняются для $p, q, r, s \in \mathbb{N}_{1, n}$ только при $q=r . \mathrm{B}$ случае $\operatorname{Tr} \sigma \cap \operatorname{Tr} \rho=\varnothing$ для любых $i \in \operatorname{Tr} \sigma$ и $j \in$ $\operatorname{Tr} \rho$, очевидно, имеет место $i \neq j$, что влечет выполнение равенств $E_{i, i} \cdot E_{j, \rho(j)}=O$ и $E_{i, i} \cdot E_{j, j}=O$. По той же причине должны выполняться равенства $E_{i, \sigma(i)} \cdot E_{j, \rho(j)}=O$ и $E_{i, \sigma(i)} \cdot E_{j, j}=O$ в случае $\sigma(i) \neq j$. Следовательно, для завершения доказательства леммы 1 осталось убедиться в том, что $\sigma(i) \neq j$ при $i \in \operatorname{Tr} \sigma$ и $j \in \operatorname{Tr} \rho$ в случае $\operatorname{Tr} \sigma \cap \operatorname{Tr} \rho=\varnothing$.

Предположим справедливость противоположного утверждения, т.е. существование таких $i^{\prime} \in \operatorname{Tr} \sigma$ и $j^{\prime} \in \operatorname{Tr} \rho$, что $\sigma\left(i^{\prime}\right)=j^{\prime}$. Тогда из $j^{\prime} \in \operatorname{Tr} \rho, \operatorname{Tr} \sigma \cap \operatorname{Tr} \rho=\varnothing$ и $(1)$ имеем включение $j^{\prime} \in \operatorname{Fix} \sigma$, т.е. равенство $\sigma\left(j^{\prime}\right)=j^{\prime}$. Из последнего с учетом $\sigma\left(i^{\prime}\right)=j^{\prime}$ следует равенство $\sigma\left(i^{\prime}\right)=\sigma\left(j^{\prime}\right)$, которое, очевидно, равносильно $i^{\prime}=j^{\prime}$. $\mathrm{C}$ другой стороны, из $i^{\prime} \in \operatorname{Tr} \sigma$ и $\sigma\left(i^{\prime}\right)=j^{\prime}$ следует справедливость соотношений $i^{\prime} \neq \sigma\left(i^{\prime}\right)=j^{\prime}$, что влечет $i^{\prime} \neq j^{\prime}$. Полученное противоречие завершает доказательство леммы 1 .

Пусть $\mathbb{N}_{1 r}=\{1,2, \ldots, r\}$ и $\mathscr{J}_{r}-$ семейство непустых подмножеств из $\mathbb{N}_{1 r}$. Следующее утверждение описывает свойство матриц подстановок, которое понадобится при доказательстве основного результата этого пункта.

ЛЕмма 2. Пусть $\varepsilon, \sigma_{1}, \sigma_{2}, \ldots, \sigma_{r} \in S_{n}$, где $\varepsilon-$ тождественная подстановка, $\sigma=\sigma_{r} \circ \sigma_{r-1} \circ \cdots \circ \sigma_{2} \circ \sigma_{1} u r \geqslant 2$. Тогда для соответствующих матрии, имеет место разложение

$$
\bar{\sigma}-\bar{\varepsilon}=\sum_{J \in \mathscr{J}_{r}} \prod_{j \in J}\left(\bar{\sigma}_{j}-\bar{\varepsilon}\right) .
$$

ДокАЗАТЕЛЬСтво. При $r=2$ лемма 2 очевидна. Действительно, в этом случае $\sigma=\sigma_{2} \circ \sigma_{1}$, откуда с учетом первого равенства (4) имеем $\bar{\sigma}=\bar{\sigma}_{1} \cdot \bar{\sigma}_{2}$ и, как показывает проверка,

$$
\bar{\sigma}-\bar{\varepsilon}=\bar{\sigma}_{1} \cdot \bar{\sigma}_{2}-\bar{\varepsilon}=\left(\bar{\sigma}_{1}-\bar{\varepsilon}\right) \cdot\left(\bar{\sigma}_{2}-\bar{\varepsilon}\right)+\left(\bar{\sigma}_{1}-\bar{\varepsilon}\right)+\left(\bar{\sigma}_{2}-\bar{\varepsilon}\right) .
$$

Далее доказательство леммы проводится индукцией по $r$. Предположим, что требуемое разложение имеет место для $\bar{\rho}=\bar{\sigma}_{1} \cdot \bar{\sigma}_{2} \cdots \bar{\sigma}_{r-2} \cdot \bar{\sigma}_{r-1}$ и убедимся в наличии такого разложения для $\bar{\sigma}=\bar{\rho} \cdot \bar{\sigma}_{r}$. По индуктивному предположению имеем разложение

$$
\bar{\rho}-\bar{\varepsilon}=\sum_{J \in \mathscr{J}_{r-1}} \prod_{j \in J}\left(\bar{\sigma}_{j}-\bar{\varepsilon}\right),
$$


где $\mathscr{J}_{r-1}$ - семейство непустых подмножеств множества $\mathbb{N}_{1, r-1}$. Из равенства $\bar{\sigma}=$ $\bar{\rho} \cdot \bar{\sigma}_{r}$ и указанного для матрицы $\bar{\rho}-\bar{\varepsilon}$ разложения следует цепочка равенств

$$
\begin{aligned}
\bar{\sigma}-\bar{\varepsilon} & =(\bar{\rho}-\bar{\varepsilon}) \cdot\left(\bar{\sigma}_{r}-\bar{\varepsilon}\right)+(\bar{\rho}-\bar{\varepsilon})+\left(\bar{\sigma}_{r}-\bar{\varepsilon}\right)=\left(\sum_{J \in \mathscr{J}_{r-1}} \prod_{j \in J}\left(\bar{\sigma}_{j}-\bar{\varepsilon}\right)\right) \cdot\left(\bar{\sigma}_{r}-\bar{\varepsilon}\right) \\
& =\sum_{J \in \mathscr{J}_{r-1}} \prod_{j \in J \cup\{r\}}\left(\bar{\sigma}_{j}-\bar{\varepsilon}\right)+\sum_{J \in \mathscr{J}_{r-1}} \prod_{j \in J}\left(\bar{\sigma}_{j}-\bar{\varepsilon}\right)+\left(\bar{\sigma}_{r}-\bar{\varepsilon}\right)=\sum_{J \in \mathscr{J}_{r}} \prod_{j \in J}\left(\bar{\sigma}_{j}-\bar{\varepsilon}\right),
\end{aligned}
$$

так как $\mathscr{J}_{r}=\left\{J, J \cup\{r\},\{r\} \mid J \in \mathscr{J}_{r-1}\right\}$. Таким образом, лемма 2 доказана.

Ниже приводятся достаточные условия несмежности вершин из $P_{\eta^{-1} H}$.

ПрЕДЛОЖЕНИЕ 4. Пусть при заданных $H \subseteq S_{n} u \eta \in H$ вершины $\bar{v}, \bar{\varepsilon}$ полито-

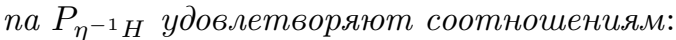

1) $\bar{v}=\bar{v}_{1} \cdot \bar{v}_{2} \cdots \bar{v}_{r}$, где $r \geqslant 2 u v_{i} \in \eta^{-1} H \backslash\{v, \varepsilon\}$ для всех $i \in \mathbb{N}_{1, r}$;

2) $\left(\bar{v}_{i}-\bar{\varepsilon}\right) \cdot\left(\bar{v}_{j}-\bar{\varepsilon}\right)=O$ для всех $i \neq j \in \mathbb{N}_{1, r}$, где $O-$ нулевая матрица.

Тогда $\bar{v}$ и $\bar{\varepsilon}-$ несмежные вершины политопа $P_{\eta^{-1} H}$.

ДокАЗАТЕЛЬСТво. Из леммы 2 и пункта 2) предложения 4 следует

$$
\bar{v}-\bar{\varepsilon}=\sum_{J \in \mathscr{J}_{r}} \prod_{j \in J}\left(\bar{v}_{j}-\bar{\varepsilon}\right)=\sum_{i \in \mathbb{N}_{1, r}}\left(\bar{v}_{i}-\bar{\varepsilon}\right),
$$

так как произведения $\prod_{j \in J}\left(\bar{v}_{j}-\bar{\varepsilon}\right)$ равны нулевой матрице для подмножеств $J \subseteq$ $\mathbb{N}_{1, r}$ мощности $|J| \geqslant 2$. Следовательно, $\bar{v}-\bar{\varepsilon}$ является конической комбинацией матриц $\bar{v}_{i}-\bar{\varepsilon}$, где $v_{i} \in \eta^{-1} H \backslash\{v, \varepsilon\}, i \in \mathbb{N}_{1, r}$, откуда с учетом следствия 1 вытекает смежность вершин $\bar{v}$ и $\bar{\varepsilon}$ политопа $P_{\eta^{-1} H}$.

Из предложения 4 и леммы 1 непосредственно вытекает

СЛЕДСТвиЕ 2. Пусть $H \subseteq S_{n}, \eta \in H u v \in \eta^{-1} H-$ произведение не менее двух подстановок из $\eta^{-1} H \backslash\{v, \varepsilon\}$, носители которых попарно не пересекаются. Тогда $\bar{v} u \bar{\varepsilon}-$ несмежсные вершины политопа $P_{\eta^{-1} H}$.

6. Критерий несмежности вершин и его следствия. Зафиксируем в $S_{n}$ произвольное подмножество $H$, выберем подстановку $\eta \in H$ и введем понятие допустимой матрицы по отношению к подстановке $v_{0} \in \eta^{-1} H$. Пусть $\Omega_{v_{0}}=\left\{\omega_{i} \mid i \in\right.$ $\left.\mathbb{N}_{1, m}\right\}$ - множество независимых циклов подстановки $v_{0}$. Тогда $(k \times m)$-матрица $A=\left(a_{i, j}\right)$, где $2 \leqslant k \leqslant 2^{m}-2$ и $2 \leqslant m \leqslant\lfloor n / 2\rfloor$, называется допустимой по отношению $\kappa v_{0}$, если выполняются следующие условия:

1) существует покрытие $J_{1}, J_{2}, \ldots, J_{k}$ множества $\mathbb{N}_{1, m}$ такое, что для любого подмножества $J_{i}$ имеет место $\prod_{j \in J_{i}} \omega_{j} \in \eta^{-1} H$, где $i \in \mathbb{N}_{1, k}$;

2) $i$-я строка матрицы $A=\left(a_{i, j}\right)$ совпадает с характеристическим вектором подмножества $J_{i}$, т.е. $a_{i, j}=1$, если $j \in J_{i}$, и $a_{i, j}=0$, если $j \notin J_{i}$, где $i \in \mathbb{N}_{1, k}$ и $j \in \mathbb{N}_{1, m}$

3) бинарная система линейных уравнений $x A=1$ совместна при дополнительном ограничении $x \geqslant 0$, где $x-k$-мерный вектор.

Ниже формулируется основной результат данной статьи, а именно, необходимые и достаточные условия несмежности вершин $\bar{v}_{0}, \bar{\varepsilon}$ политопа $P_{\eta^{-1} H}$. 
Tеорема 3. При заданных $H \subseteq S_{n} u \eta \in H$ вершины $\bar{v}_{0}$ u $\bar{\varepsilon}$ nолитопа $P_{\eta^{-1} H}$ несмежни тогда и только тогда, когда для подстановки $v_{0}$ существует допустимая $(k \times m)$-матрица $A=\left(a_{i, j}\right)$.

ДоКАЗАТЕЛЬСтво. Убедимся в необходимости данных условий. Для этого выберем в $P_{\eta^{-1} H}$ несмежные вершины $\bar{v}_{0}, \bar{\varepsilon}$, где $v_{0}$ - подстановка с множеством независимых циклов $\Omega_{v_{0}}=\left\{\omega_{i} \mid i \in \mathbb{N}_{1, m}\right\}$, и построим для $v_{0}$ допустимую матрицу $A=\left(a_{i, j}\right)$.

Так как для любой подстановки число независимых циклов в разложении вида (2) не превосходит $\lfloor n / 2\rfloor$, то $m \leqslant\lfloor n / 2\rfloor$. Далее, в силу следствия 1 и предложения 3 для $\bar{v}_{0}-\bar{\varepsilon}$ выполняется матричное равенство

$$
\bar{v}_{0}-\bar{\varepsilon}=\sum_{v \in \Upsilon} \lambda_{v}(\bar{v}-\bar{\varepsilon}),
$$

в котором $0<\lambda_{v} \in \mathbb{R}, \Upsilon \subseteq \eta^{-1} H \backslash\left\{v_{0}, \varepsilon\right\},|\Upsilon| \geqslant 2$ и любая подстановка $v \in \Upsilon$ определяется некоторым подмножеством $J_{v} \subset \mathbb{N}_{1, m}$, т.е.

$$
v=\prod_{j \in J v} \omega_{j}
$$

Так как $|\Upsilon| \geqslant 2$, то $m \geqslant 2$ (в противном случае при $m=1$ подмножество $\Upsilon$ может включать только один цикл $\omega_{1}$, который совпадает с $v_{0}$, что влечет $\left.\Upsilon=\varnothing\right)$. Следовательно, $2 \leqslant m \leqslant\lfloor n / 2\rfloor$ и подмножество подстановок $\Upsilon$ определяется некоторым набором собственных подмножеств $J_{1}, J_{2}, \ldots, J_{k} \subset \mathbb{N}_{1, m}$, где $2 \leqslant k \leqslant 2^{m}-2$, т.е. $\Upsilon$ состоит из подстановок

$$
v_{i}=\prod_{j \in J_{i}} \omega_{j}, \quad \text { где } \quad i \in \mathbb{N}_{1, k}, \quad \omega_{j} \in \Omega_{v_{0}} .
$$

Из первого равенства (4) независимости циклов, входящих в $\Omega_{v_{0}}$, и полученных разложений для подстановок из $\Upsilon$, следует, что соответствующие им матрицы имеют вид

$$
\bar{v}_{i}=\prod_{j \in J_{i}} \bar{\omega}_{j}, \quad \text { где } \quad i \in \mathbb{N}_{1, k} .
$$

Следовательно, коническое разложение матрицы $\bar{v}_{0}-\bar{\varepsilon}$ с учетом

$$
\bar{v}_{0}=\prod_{j \in \mathbb{N}_{1, m}} \bar{\omega}_{j}
$$

имеет вид

$$
\prod_{j=1}^{m} \bar{\omega}_{j}-\bar{\varepsilon}=\sum_{i=1}^{k} \lambda_{i}\left(\prod_{j \in J_{i}} \bar{\omega}_{i}-\bar{\varepsilon}\right) .
$$

Так как циклы $\omega_{i} \in \Omega_{v_{0}}$ независимы, то $\operatorname{Tr} \omega_{i} \cap \operatorname{Tr} \omega_{j}=\varnothing$ для всех $i \neq j$ из $\mathbb{N}_{1, m}$, откуда с учетом лемм 1, 2 следует справедливость равенств

$$
\prod_{j=1}^{m} \bar{\omega}_{j}-\bar{\varepsilon}=\sum_{j=1}^{m}\left(\bar{\omega}_{j}-\bar{\varepsilon}\right), \quad \prod_{j \in J_{i}} \bar{\omega}_{j}-\bar{\varepsilon}=\sum_{j \in J_{i}}\left(\bar{\omega}_{j}-\bar{\varepsilon}\right) .
$$


Пусть $\left(a_{i, 1}, a_{i, 2}, \ldots, a_{i, m}\right)$ - характеристический вектор подмножества $J_{i}$. Тогда второе равенство (9) равносильно

$$
\prod_{j \in J_{i}} \bar{\omega}_{j}-\bar{\varepsilon}=\sum_{j=1}^{m} a_{i, j}\left(\bar{\omega}_{j}-\bar{\varepsilon}\right), \quad \text { где } \quad i \in \mathbb{N}_{1, k} .
$$

Подставляя найденные выражения для матриц $\prod_{j=1}^{m} \bar{\omega}_{j}-\bar{\varepsilon}$ и $\prod_{j \in J_{i}} \bar{\omega}_{j}-\bar{\varepsilon}$ в (8) и проведя преобразования, получим цепочку равенств

$$
\begin{gathered}
\sum_{j=1}^{m}\left(\bar{\omega}_{j}-\bar{\varepsilon}\right)=\sum_{j=1}^{k} \lambda_{i}\left(\sum_{j=1}^{m} a_{i, j}\left(\bar{\omega}_{j}-\bar{\varepsilon}\right)\right) \\
\Longleftrightarrow \sum_{j=1}^{m}\left(\bar{\omega}_{j}-\bar{\varepsilon}\right)=\sum_{j=1}^{m} \sum_{i=1}^{k} \lambda_{i} a_{i, j}\left(\bar{\omega}_{j}-\bar{\varepsilon}\right) \\
\Longleftrightarrow \sum_{j=1}^{m}\left(\bar{\omega}_{j}-\bar{\varepsilon}\right)=\sum_{j=1}^{m}\left(\sum_{i=1}^{k} \lambda_{i} a_{i, j}\right)\left(\bar{\omega}_{j}-\bar{\varepsilon}\right) \\
\Longleftrightarrow \sum_{j=1}^{m}\left(\sum_{i=1}^{k} \lambda_{i} a_{i, j}-1\right)\left(\bar{\omega}_{j}-\bar{\varepsilon}\right)=O,
\end{gathered}
$$

где $O$ - нулевая матрица. Так как циклы $\omega_{j}$, где $j \in \mathbb{N}_{1, m}$, независимы, что влечет $\operatorname{Tr} \omega_{i} \cap \operatorname{Tr} \omega_{j}=\varnothing$ при $i \neq j \in \mathbb{N}_{1, m}$, то в силу леммы 1 скалярные произведения $\left(\bar{\omega}_{i}-\bar{\varepsilon}, \bar{\omega}_{j}-\bar{\varepsilon}\right)$ равны нулю для всех $i \neq j \in \mathbb{N}_{1, m}$. Таким образом, матрицы $\bar{\omega}_{j}-\bar{\varepsilon}$, где $j \in \mathbb{N}_{1, m}$, попарно ортогональны, что влечет их линейную независимость и, следовательно, равенство нулю всех коэффициентов последнего матричного равенства из (10), т.е. справедливость числовых равенств

$$
\sum_{i=1}^{k} \lambda_{i} a_{i, j}=1, \quad \text { где } i \in \mathbb{N}_{1, k} .
$$

Следствием последних является совместность бинарной системы линейных уравнений $x A=1$ при ограничении $x \geqslant 0$, так как $0<\lambda_{i} \in \mathbb{R}$ для всех $i \in \mathbb{N}_{1, k}$. Так как $i$-й строкой матрицы $A$ является характеристический вектор подмножества $J_{i}$, обладающего свойством

$$
\prod_{j \in J_{i}} \omega_{j} \in \Upsilon \subseteq \eta^{-1} H \backslash\left\{v_{0}, \varepsilon\right\}, \quad \text { где } \quad i \in \mathbb{N}_{1, k},
$$

то $(k \times m)$-матрица $A=\left(a_{i, j}\right)$ является допустимой матрицей к подстановке $v_{0}$.

Покажем, что рассматриваемые условия достаточны, т.е. обеспечивают смежность вершин $\bar{v}_{0}, \bar{\varepsilon}$ политопа $P_{\eta^{-1}}$. Пусть для подстановки $v_{0}$ с множеством независимых циклов $\Omega_{v_{0}}=\left\{\omega_{j} \mid j \in \mathbb{N}_{1, m}\right\}$ существует допустимая $(k \times m)$-матрица $A=\left(a_{i, j}\right)$. Тогда $2 \leqslant m \leqslant\lfloor n / 2\rfloor, 2 \leqslant k \leqslant 2^{m}-2$ и в силу совместности системы уравнений $x A=1$ при $x \geqslant 0$ должен существовать такой набор чисел $0 \leqslant \lambda_{i} \in \mathbb{R}$, где $i \in \mathbb{N}_{1, k}$, что

$$
\sum_{i=1}^{k} \lambda_{i} a_{i, j}-1=0
$$


для всех $j \in \mathbb{N}_{1, m}$. Следовательно, должно выполняться матричное равенство

$$
\sum_{j=1}^{m}\left(\sum_{i=1}^{k} \lambda_{i} a_{i, j}-1\right)\left(\bar{\omega}_{j}-\bar{\varepsilon}\right)=O,
$$

которое в силу (10) равносильно равенству

$$
\sum_{j=1}^{m}\left(\bar{\omega}_{j}-\bar{\varepsilon}\right)=\sum_{i=1}^{k} \lambda_{i}\left(\sum_{j=1}^{m} a_{i, j}\left(\bar{\omega}_{j}-\bar{\varepsilon}\right)\right) .
$$

Так как $A=\left(a_{i, j}\right)$ допустимая, то ее $i$-я строка $\left(a_{i, 1}, a_{i, 2}, \ldots, a_{i, m}\right)$ - характеристический вектор подмножества $J_{i} \subset \mathbb{N}_{1, m}$, где $i \in \mathbb{N}_{1, k}$. Следовательно, имеет место равенство

$$
\sum_{j=1}^{m}\left(\bar{\omega}_{j}-\bar{\varepsilon}\right)=\sum_{i=1}^{k} \lambda_{i}\left(\sum_{j \in J_{i}}\left(\bar{\omega}_{j}-\bar{\varepsilon}\right)\right) .
$$

В силу независимости циклов из $\Omega_{v_{0}}$ выполняются равенства $(9)$, из которых и последнего равенства следует равенство

$$
\prod_{j=1}^{m} \bar{\omega}_{j}-\bar{\varepsilon}=\sum_{i=1}^{k} \lambda_{i}\left(\prod_{j \in J_{i}} \bar{\omega}_{j}-\bar{\varepsilon}\right),
$$

которое равносильно

$$
\bar{v}_{0}-\bar{\varepsilon}=\sum_{i=1}^{k} \lambda_{i}\left(\bar{v}_{i}-\bar{\varepsilon}\right),
$$

где $0 \leqslant \lambda_{i} \in \mathbb{R}$ и подстановки вида $v_{i}=\prod_{j \in J_{i}} \omega_{j}$ принадлежат $\eta^{-1} H \backslash\left\{v_{0}, \varepsilon\right\}$ в силу допустимости матрицы $A$. Так как не все $\lambda_{i}$ равны нулю (в противном случае нулевой вектор являлся бы решением системы уравнений $x A=1$, что невозможно), то, оставляя в правой части последнего матричного равенства только ненулевые слагаемые, будем иметь требуемое следствием 1 коническое разложение матрицы $\bar{v}_{0}-\bar{\varepsilon}$, из которого следует несмежность вершин $\bar{v}_{0}$ и $\bar{\varepsilon}$ политопа $P_{\eta^{-1} H}$. Теорема 3 доказана.

Так как для любых вершин $\bar{v}_{0}, \bar{\varepsilon}$ политопа $P_{\eta^{-1} H}$ возможны только две альтернативы: либо они смежны, либо не смежны, - то отрицание условий теоремы 3 дает критерий смежности указанной пары вершин.

СлеДСТВИЕ 3. При заданных $H \subseteq S_{n} u \eta \in H$ вершины $\bar{v}_{0} u \bar{\varepsilon}$ nолитопа $P_{\eta^{-1} H}$ являются смежными тогда и только тогда, когда для подстановки $v_{0}$ не существует допустимой матриць $A$.

Теоремы 1, 3 и следствие 3 позволяют сформулировать критерий как смежности, так и несмежности вершин $\eta, v$ политопа $P_{H}$, порожденного произвольным подмножеством $H$ симметрической группы $S_{n}$.

СЛЕДСТвиЕ 4. При заданном $H \subseteq S_{n}$ вершины $\bar{\eta} u \bar{v}$ nолитопа $P_{H}$ являются несмежными (смежсыми) тогда и толъко тогда, когда для подстановки $\eta^{-1} \circ v$ существует допустимая матрица $A$ (не существует допустимой матрицы $A$ ). 
Сформулированные Хэллером [5] и значительно позже Подбергом и Рао [4] критерии смежности соответственно для пар вершин $\bar{\varepsilon}, \bar{v}_{0}$ и $\bar{\eta}, \bar{v}$ политопа задачи о назначениях $P_{S_{n}}$ являются прямыми следствиями предложенных новых условий смежности.

ПреДЛОЖЕНИЕ 5 (Хэллер [5]). Если $\bar{\varepsilon}$ - вершина политопа $P_{S_{n}}$, coответствующая тождественной подстановке $\varepsilon$, то множество всех смежных $c \bar{\varepsilon}$ вершин из $P_{S_{n}}$ совпадает в точности с множеством вершин, соответствующих ицклам длины, большей единицы.

ДокАЗАТЕЛЬСтво. Для произвольного цикла $\omega$ длины большей 1 , очевидно, не существует допустимой матрицы, так как в этом случае $m=1$. Следовательно, в силу следствия 3 вершина $\bar{\omega}$ политопа $P_{S_{n}}$ смежна с $\bar{\varepsilon}$. Далее, простая проверка показывает, что для любой подстановки $v_{0}$, представимой в виде произведения $m \geqslant 2$ независимых циклов, единичная матрица порядка $m$ является допустимой, что влечет в силу теоремы 3 несмежность вершин $\bar{v}_{0}$ и $\bar{\varepsilon}$ политопа $P_{S_{n}}$, т.е. справедливость предложения 5 доказана.

ПреДЛОЖениЕ 6 (Падберг-Рао [4]). Две различные вериины $\bar{\eta}, \bar{v}$ nолитопа $P_{S_{n}}$ являются смежными тогда и только тогда, когда существует такая подстанов$\kappa a \omega$, что $v=\eta \circ \omega$, и состоит из единственного иикла длинъ, большей $1, u$, возможно, некоторых циклов длины 1.

ДоказАтельство. В силу теоремы 1 и равенства $\eta^{-1} S_{n}=S_{n}$ вершины $\bar{\eta}$ и $\bar{v}$ политопа $P_{S_{n}}$ являются смежными тогда и только тогда, когда смежны вершины $\bar{\varepsilon}$ и $\bar{v} \cdot \bar{\eta}^{-1}$ политопа $P_{\eta^{-1} S_{n}}=P_{S_{n}}$. Из предложения 5 следует, что $\bar{\varepsilon}$ и $\bar{v} \cdot \bar{\eta}^{-1}$ смежны тогда и только тогда, когда подстановка $\eta^{-1} \circ v-$ цикл неединичной длины. Таким образом, предложение 6 доказано.

Предложенные критерии позволяют алгоритмически решать задачу распознавания (не)смежности произвольной пары вершин $\bar{v}, \bar{\eta}$ политопа $P_{H}$, порожденного произвольным подмножеством подстановок $H$. Общая схема алгоритма состоит из следующих этапов.

Этап 1. Найти независимые циклы $\omega_{j}$, где $j \in \mathbb{N}_{1, m}$, подстановки $\eta^{-1} \circ v$, где $\eta$ и $v$ определяются выбранными вершинами $\bar{\eta}$ и $\bar{v}$ политопа $P_{H}$.

Этап 2. В случае $m \geqslant 2$ (в противном случае вершины $\bar{v}, \bar{\eta}$ смежны) вычислить бинарную матрицу $A=\left(a_{i, j}\right), i$-я строка которой является характеристическим вектором $i$-го по счету собственного подмножества $J_{i} \subset \mathbb{N}_{1, m}$ такого, что подстановка $\prod_{j \in J_{i}} \omega_{j}$ принадлежит множеству $\eta^{-1} H$.

Этап 3. Определить, совместна ли смешанная линейная система вида

$$
\left\{\sum_{i=1}^{k} a_{i, j} x_{i}=1, x_{i} \geqslant 0 \mid j \in \mathbb{N}_{1, m}\right\},
$$

где $2 \leqslant k \leqslant 2^{m}-2$ - число собственных подмножеств, вычисленных на этапе 2 .

Из описания схемы видно, что наиболее трудоемкими являются два последних этапа, так как время выполнения этапа 1 составляет $O\left(n \log _{2} n\right)$ битовых операций. Если затраты на проверку принадлежности подстановки $\prod_{j \in J_{i}} \omega_{j}$ множеству $\eta^{-1} H$ считать также равными $O\left(n \log _{2} n\right)$, то нетрудно проверить, что этап 2 включает $O\left(\left(2^{m}-2\right) n^{2} \log _{2}^{2} n\right)$ битовых операций, где $2 \leqslant m \leqslant\lfloor n / 2\rfloor-$ число независимых 
циклов подстановки $\eta^{-1} \circ v$ в разложении вида (2). Трудоемкость этапа 3 , очевидно, определяется временем, затрачиваемым на выявление совместности бинарной системы линейных уравнений вида $x A=1$ при ограничении $x \geqslant 0$. Указанная система включает $m$ уравнений, зависящих от $k$ переменных, где $2 \leqslant k \leqslant 2^{m}-2$.

Согласно [20; с. 297] определение совместности смешанной системы

$$
\left\{\sum_{i=1}^{k} a_{i, j} x_{i}=1, x_{i} \geqslant 0 \mid j \in \mathbb{N}_{1, m}\right\}
$$

методом Кармаркара требует $O\left(k^{4 / 5} \log _{2} k\right)$ операций над $O\left(k \log _{2} k\right)$ разрядными числами, что равносильно выполнению $O\left(k^{9 / 5} \log _{2}^{2} k\right)$ битовых операций. Следовательно, с учетом неравенства $k \leqslant 2^{m}-2$ число битовых операций этапа 3 не превосходит $O\left(\left(2^{m}-2\right)^{9 / 5} m^{2}\right)$, а общее время выполнения предложенной алгоритмической схемы составляет $O\left(\left(2^{m}-2\right)^{9 / 5} m^{2}+\left(2^{m}-2\right) n^{2} \log _{2}^{2} n+n \log _{2} n\right)$. Таким образом, в случае $m=O(1)$ эта схема имеет полиномиальную от $n$ трудоемкость для любого фиксированного подмножества $H$ симметрической группы $S_{n}$.

\section{СПИСОК ЦИТИРОВАННОЙ ЛИТЕРАТУРЫ}

[1] B. Grünbaum, Convex Polytopes, Wiley, London, 1967.

[2] D. Hausmann, Adjacency on Polytopes in Combinatorial Optimization, Math. Systems in Economics, 49, Meisenheim am Glan, Haim, 1980.

[3] M.L. Balinski, A. Russakoff, "On the assignment polytope", SIAM Rev., 16:4 (1974), $516-525$.

[4] M. W. Padberg, M. R. Rao, "The travelling salesman problem and a class of polyhedra of diameter two", Math. Programming, 7 (1974), 32-45.

[5] I. Heller, "On the traveling salesman problem", Proc. Second Symp. Linear Programming, 2, Washington, 1955, 643-665.

[6] I. Heller, "Neighbor relations on the convex of cyclic permutations", Pacific J. Math., 6:3 (1956), 467-477.

[7] E. L. Lawler, J. K. Lenstra, A. H. G. Rinnooy Kan, D. B. Shmoys, The Traveling Salesman Problem, Wiley, Chichester, 1985.

[8] G. Gutin, A.P. Punnen, The Traveling Salesman Problem and Its Variations, Kluwer, Dordrecht, 2002.

[9] K. G. Murty, "On the tours of a traveling salesman", SIAM J. Control. Optim., 7:1 (1969), $122-131$.

[10] M. R. Rao, "Adjacency of the traveling salesman tours and 0-1 vertices", SIAM J. Appl. Math., 30:2 (1976), 191-198.

[11] E. Balas, M.W. Padberg, "Adjacent vertices of the all $0-1$ programming polytope", RAIRO Rech. Opér., 13:1 (1979), 3-12.

[12] В.И. Сарванов, О многогранниках, связанных с оптимизацией на подстановках, Препринт № 7 (23), Ин-т матем. АН БССР, Минск, 1977.

[13] В. М. Демиденко, В.А. Шлык, "О смежности вершин многогранников задач комбинаторной оптимизации”, Докл. АН БССР, 33:9 (1989), 773-776.

[14] В. М. Демиденко, "Условия сильной разрешимости оптимизационных задач на подстановках и их применение", Докл. НАН Беларуси, 45:4 (2001), 9-12.

[15] C. H. Papadimitriou, "The adjacency relation on the traveling salesman polytope is NPcomplete", Math. Programming, 14:3 (1978), 312-324.

[16] В. М. Демиденко, Критерий смежности вершин на выпуклой оболочке матрии подстановок (многогранник задачи о коммивояжере), Препринт № 28 (338), Ин-т матем. АН БССР, Минск, 1988. 
[17] V.M. Demidenko, "Vertices adjacency criterion for polytope of the traveling salesman problem", Proceeding of the International Conference on Operation Research (ETH Zürich, 31 August-3 September 1998), Annual Meeting: GOR, ÖGOR, SIGOPT, Zürich, 1998, $54-55$.

[18] Д. А. Супруненко, Группы подстановок, Навука і тэхніка, Минск, 1996.

[19] В. А. Емеличев, М. М. Ковалев, М. К. Кравцов, Многогранники, графы, оптимизаиия (комбинаторная теория многогранников), Наука, М., 1981.

[20] А. Схрейвер, Теория линейного и челочисленного программирования, 1, Мир, М., 1991.

В. М. Демиденко

Поступило

Институт математики НАН Беларуси, г. Минск

03.05.2005

E-mail: demidenko@bas-net.by

Исправленный вариант

12.12 .2005 